\section{Orientation Effects in Solid Polymers}

The 5th Europhysics Conference on Macromolecular Physics in Budapest (27-30 April) was attended by about 175 participants including 50 from Hungary, 40 from other countries in Eastern Europe and 85 from Western Europe and the U.S.A.

The subject "Orientation Effects in Solid Polymers" appeared well chosen because it served as a link between experimentalists and theoreticians in various fields as well as between fundamental studies and their industrial applications. The main subject was divided into five topics:

I. Methods of Characterisation and Measurement of Molecular Orientation,

II. Mechanical and Physical Properties of Uni- and Biaxially Oriented Films,

III. Structure and Properties of Highly Oriented Fibers,

IV. Properties of Block Copolymers

V. Crystallisation Induced by Shear or Elongational Flow.

Although the relevance to technological properties formed the background and sometimes the foreground to some of the lectures, most of the studies reported in this conference were of a fundamental nature.

Under topic V., for instance, there were not only the beautiful experimental studies of Pennings, of Keller and of Yeh on Shear and Strain Induced Crystallisation, but also more theoretical papers by Ziabicki on the thermodynamics and on the mechanism of crystallisation.

Clearly, macromolecules offer promising possibilities for the study of the thermodynamics and kinetics of crystallisation in general and also for the production of crystals of very different, and sometimes exceptional, properties by suitable strain-temperature treatments.

In many respects polymer physics can be compared to solid state physics, both in the interaction between fundamental and applied science and in the fact that subtle structural changes induce large effects on measurable and technological properties.

Apart from 32 communications each of 15 minutes, for every topic there were one or more introductory papers which were generally of high quality.

Some of the main lectures (Ward, May, Yeh and Keller) and most of the short communications are printed in Volume I D of Europhysics Conference Abstracts.

\section{A.J. Staverman}

The Dept. of Physics of the Rijksuniversiteit Groningen has a vacancy for a :

\title{
LECTOR IN MATERIALS SCIENCE
}

(lector is similar to reader or associate professor)

The lector will be teaching materials science and physical metallurgy at the undergraduate and graduate levels, covering both the wide scope of problems in materials science and the physical basis of physical metallurgy. He will carry out his research in the Physical Metallurgy Laboratory and will be expected to coach and guide diploma and Ph. D. students. The main research topics in the Laboratory are: crystal plasticity and dislocation dynamics, order-disorder phenomena, electron microscopy, computer simulations. The Dept. is looking for a solid-state physicist or -chemist, with a record of active interest in materials science and the physics of defects in the solid state, one who derives inspiration from problems in materials science and tries to solve them in terms of quantitative physical models by judicious experimental or theoretical investigation.

Interested persons should contact the secretary of the appointment committee

Prof. A. W. Sleeswyk, Laboratorium voor Fysische Metaalkunde,

Nijenborgh 18, The Netherlands. (Tel. : 050 - 115 929)

Applicants are requested to submit with their application, a curriculum vitae and a list of publications, not later than 1 October 1976.

\section{Quantum Electronics Approaching Maturity}

The IXth International Quantum Electronics Conference held in Amsterdam, June 14-18, with which was associated a laser exhibition, was attended by 850 scientists ; 171 contributed, 49 invited and 46 post-deadline papers were presented. As was to be expected after more than 15 years of research in quantum electronics, a large number of papers were devoted to technical progress and refinements of existing methods and devices.

The whole visible and a good deal of the infra red and ultra violet spectrum is now covered by continuously tunable dye lasers and/or nonlinearmixing processes. Except for the shortest wavelengths, radiation is available either as continuous wave at low and medium intensities (say $<1 \mathrm{~W}$ ) or pulsed with intensities in the $\mathrm{kW}$ to $\mathrm{MW}$ range, ps to ns duration, and repetition rates of typically $1-100 \mathrm{~s}^{-1}$. The short wavelength limit of coherent radiation has sucessfully been pushed down from previously $887 \AA$ to $570 \AA$ by means of frequency tripling of a $\mathrm{Xe}$, laser. Considerable progress has been made in the ultra violet and vacuum ultra violet with noble gashalogen mixtures, pumped with electron guns and electrical discharge excited noble gas halides. It has turned out, on the other hand, that lasing at X-ray frequencies is extremely difficult to achieve and will require further hard and sophisticated work.

Stable tunable lasers are now widely used for high-resolution spectroscopy and this has led in particular to a substantially broadened knowledge of the fine structure in the spectra of gases, improved time standards and fundamental constants (Rydberg frequency). A breakthrough in the spectroscopy of larger molecules may be expected in the near future.

Narrow-line high power gas lasers are able to excite selectively only one isotopic species of a gas which allows for the subsequent isotope separation of various elements. The dissociation of $\mathrm{UF}_{6}$, which is of particular technical interest, requires radiation around 16 $\mu \mathrm{m}$. Successful efforts have been made to find powerful lasers or nonlinear mixing schemes for this frequency.

The proven $\mathrm{Nd}$ : glass, $\mathrm{CO}_{2}$, and iodine lasers have been further developed. Power levels of more than 1 TW have been achieved in a single amplifier chain of $\mathrm{Nd}$ : glass lasers. The construction of glass laser systems depositing 150-300 TW (100-200 kJ) on laser fusion targets is now being considered. Laser implosions with ion temperatures of $2 \mathrm{keV}$, Lawson numbers of $10^{12} \mathrm{~cm}^{3} \mathrm{~s}$ and compression ratios of $10^{2}-10^{3}$ have been achieved. Particular attention is now being paid to the interpretation of X-ray emission from laser plasmas (electron temperature, etc.) and neutron and ion yields (interaction parameters).

In nonlinear optics, exploitation of the enhancement of higher-order susceptibilities near a resonance has introduced a variety of new spectroscopy methods, and yielded numerous new data, for example natural lifetimes and dephasing times of selected excited states, transition probabilities for 


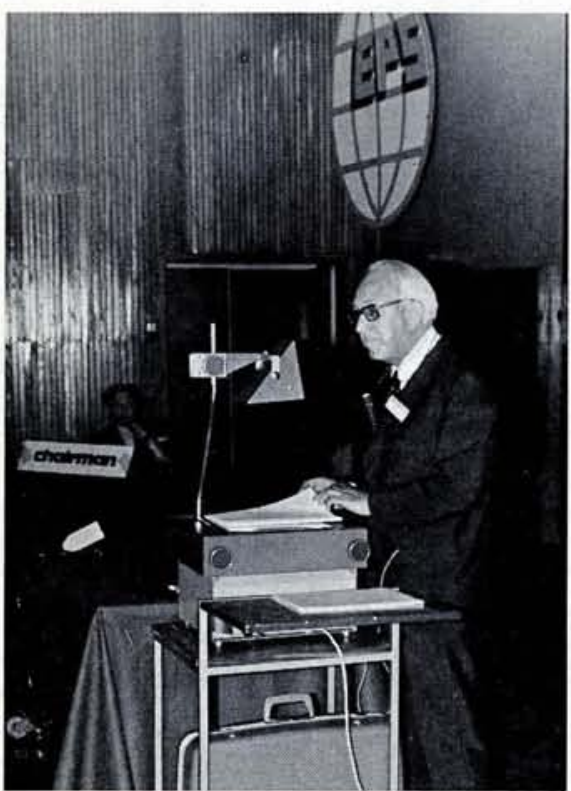

Quantum electronics cont.

multiphoton absorption, coupling constants for near-resonant processes (Raman, etc.) induced birefringence, optical activity and many more. This work at present concentrates on gases being particularly "clean systems" but imporiant experiments have been made also with liquids and solids. On the other hand, nonlinear mixing becomes an increasingly useful tool for the generation of new and tunable frequencies, in particular in the uv and ir.

The availability of pulsed transformlimited tunable dye lasers has allowed considerable progress in the experimental observation of coherent resonant phenomena including multisoliton effects and focusing when the carrier frequency is at exact resonance. Coherent multiphoton processes are now being investigated, as well as fluorescence and resonance scattering which promises to yield considerable information on collisional processes.

The translation of electrotechnical building units to the optical regime has created the subdiscipline of " optical electronics". Reports on ir antennas, distributed feedback lasers, tunnel junctions, heterodyne detection, and integrated optics gave an overview of this field.

Very interesting are the aspects of laser chemistry : detection and control of chemical reactions, for instance, the synthesis of alkalihydrides from vapour ("laser snow"), molecular dissociation (isotope separation, see above) or, very important, the interaction with biological molecules. Important contributions may be expected from this interdisciplinary field in the near future.

D. Pohl

Professor P. Brix giving the first invited lecture at the opening session chaired by the Chairman of the N.P. Division of the EPS Dr. C. Van der Leun.

\section{Radial Shape of Nuclei}

Although the 2nd Nuclear Physics Divisional Conference of the EPS held in Cracow, 22-25 June 1976, was not called so officially, it was really a jubilee conference. 65 years have passed since, in 1911, Rutherford with his collaborators discovered the radial dimensions of nuclei, 25 years from 1951 when Lyman, Hanson and Scott pushed forward our knowledge on the radii of charge distribution in nuclei using fast electrons, and 20 years since the famous Hofstadter paper was published in 1956 in Rev. Mod. Phys.

Organized by the Institute of Physics of the Jagellonian University and the Cracow Institute of Nuclear Physics under the chairmanship of Professor A. Budzanowski the Cracow Conference, summarized our understanding of nuclear charge and matter distributions, especially since the International Conference on Electromagnetic Sizes of Nuclei, 1967 in Ottawa, the last conference on this subject. (The First Nuclear Physics Divisional Conference of the EPS was held in Harwell, 24-26 March 1975.)

The first invited talk by P. Brix of Heidelberg illustrated well the progress made since the time when 20 years ago, Hofstadter stated in his paper that "the experiments are just scratching the surface". Now comparison of charge distributions in neighbouring isotones or isotopes gives direct experimental information on the squares of the single-particle radial wave functions of nucleons.

When penetrating deeply into the interior of nuclei we meet a fundamental problem of the validity of quantum electrodynamics at very small distances, say $10 \%$ of the radius of the nucleus, i.e. of the order of $5.10^{-15}$ $\mathrm{cm}$. R. Hofstadter in a special lecture presented the experimental details and results of the most recent Stanford $\mathrm{e}^{+}-\mathrm{e}^{-}$collision experiment proving this validity down to distances of the order of $2.10^{-15} \mathrm{~cm}$. For much deeper insight into the proton structure this limit should still be pushed down to distances of about $8.10^{-16} \mathrm{~cm}$.

Not only the distribution of charge but also of higher electric and magnetic moments of nuclei are now investigated with an increasing accuracy as it was shown in the lecture of R. Eng- fer of Zurich. Another Zurichois, H. K. Walter from SIN in Villigen presented the actual status of our knowledge of the size of nuclei in excited states but pointed out some stagnation in this field of research.

In order to extract information on radial shapes of nuclei we should not only have sufficiently powerful tools at our disposal but also be convinced of the validity of our understanding of interaction. It is also very essential to interpret the data in the proper, umambiguous way. Y.N. Kim of Texas Techn. University has shown how, using some series expansions, the information about charge and matter distribution could be represented in a way independent of some particular model for the distribution function. However, such a reprensentation is unable to solve the problem of the very deep nuclear interior. The predicted bubbles in the form of a large depression of density near the centre of some nuclei, like ${ }^{36} \mathrm{Ar}$, do not seem incompatible with the existing experimental data.

The problems of the theoretical approach to matter distribution in nuclei on the basis of many body theory were covered in the lecture of J.W. Negele of MIT. The speaker pointed out that the present status of theory seems good enough to be used as a quantitative tool in understanding strong interactions in nuclei and not only to extract information on the distribution of nucleons.

The charge distribution in nuclei is still known much better than the matter distribution. D.F. Jackson of Guildford (England) and $\mathrm{H}$. Rebel of Karlsruhe have shown that according to our present knowledge, coming from investigations of hadron interactions with nuclei, any difference between proton and neutron rms radii of nuclei is small, of the order of at most 0,2 $\mathrm{fm}$, which however, is not inconsistent with the possibility of the existence of quite large neutron excess in extreme surface.

Do clusters exist in nuclei ? V. Neudatchin of Moscow presented a theoretician's point of view on this subject, while K. Grotowski of Cracow proposed some possible experimental evidence, especially connected with so called ALAS (anomalous large angle 